\title{
Irinotecan - klinischer Fortschritt in der Second-line-Therapie des fortgeschrittenen Kolorektalkarzinoms
}

\section{Irinotecan - Clinical Progress in the Second-Line Therapy of Advanced Colorectal Cancer}

\author{
C. Bokemeyer \\ Hämatologie und Onkologie, Eberhard-Karls-UniversitätTübingen
}

\section{Einführung}

Kolorektale Karzinome stellen einen A nteil von $15 \%$ aller malignen Tumoren, ihre jährliche Inzidenz in der B evölkerung beträgt 50-60 Neuerkrankungen pro 100.000 Personen. E in $D$ rittel der Tumoren sind im R ektum lokalisiert, zwei D rittel im Kolon. In etwa $90 \%$ der Fälle handelt es sich um A denokarzinome. Aufgrund seiner großen Verbreitung besitzt das Kolorektalkarzinom auch eine große gesundheitsökonomische B edeutung. U nter diesem $G$ esichtspunkt ist es daher ebenfalls wichtig, Therapiestandards für diese E rkrankung zu etablieren. Für mehr als 30 Jahre war 5-Fluorouracil das wirksamste Zytostatikum in der Behandlung kolorektaler Karzinome. In der M onotherapie induziert es eine objektive R emissionsrate von etwa 10-20\% . Die zytotoxische Wirkung beruht auf einer $\mathrm{H}$ emmung der Thymidylat-Synthase, was zu einer Störung des Pyrimidinstoffwechsels und damit der Neusynthese von N ukleinsäuren führt. B is vor wenigen Jahren gab es kaum weitere Substanzen, die eine nennenswerte A ktivität aufwiesen. $M$ itomycin $C$ und Nitrosoharnstoff induzierten A nsprechraten von unter $10 \%$. L ange Z eit war es umstritten, ob in der metastasierten Situation überhaupt eine Chemotherapie sinnvoll sei. D iese Frage wurde erst in den 90er Jahren in randomisierten Studien geklärt. I $m$ Vergleich verschiedener 5-Fluorouracil-Schemata mit einer optimalen supportiven Therapie («best supportive care» B SC) stellte sich heraus, dass mit der Chemotherapie nicht nur die L ebensqualität der Patienten verbessert, sondern auch die mediane Ü berlebensdauer von etwa 6 auf 11 M onate verlängert wird. Z wei Wege wurden in den $90 \mathrm{er}$ Jahren eingeschlagen, um die Wirksamkeit von 5-Fluorouracil zu verbessern: Z um einen durch B iomodulation mit Folinsäure (ein Prinzip, das sich inzwischen weitgehend durchgesetzt hat), zum anderen durch eine Verlängerung der Infusionsdauer, z. B. auf 24 Stunden im A IO - oder A rdalan-Schema. Im randomisierten Vergleich wurden mit dem 48stündigen Langzeitinfusionsschema von D e G ramont signifikant höhere R emissionsraten erzielt als mit dem Bolus-Schema der M ayo Clinic [1].
A uch das progressionsfreie Intervall wurde verlängert, nicht jedoch die Ü berlebenszeit.

Inzwischen steht mit I rinotecan $\left(\right.$ Campto $\left.^{\circledR}\right)$ ein weiteres effektives Zytostatikum für die Therapie metastasierter kolorektaler Tumoren zur Verfügung. D ie U rsprungssubstanz, das Camptothecin, wurde in den 60er Jahren aus der fernöstlichen B aumart Camptotheca acuminata isoliert. I rinotecan wurde daraus durch chemische Modifizierung synthetisiert. Nachdem der außergewöhnliche Wirkmechanismus von I rinotecan erkannt worden war (es hemmt die Topoisomerase I, ein Schlüsselenzym für die R eplikation der DNA) und die ersten Berichte über die klinische Wirksamkeit beim kolorektalen Karzinom erschienen, wurde die E ntwicklung von I rinotecan in den späten 80er und zu B eginn der 90er Jahre stark forciert. Z wischen 1994 und 1996 wurde I rinotecan in Japan, Frankreich und den U SA zugelassen, 1998 auch in D eutschland für die Second-line-Therapie des fortgeschrittenen kolorektalen $\mathrm{K}$ arzinoms.

\section{Phase-II-Studien mit Irinotecan}

I rinotecan wurde bis etwa 1998 in mehreren Phase-II-Studien in der Second-line-Therapie geprüft. In den USA und E uropa wurden dabei unterschiedliche Schemata eingesetzt. In den U SA wurde I rinotecan in einer D osierung von $100-150 \mathrm{mg} / \mathrm{m}^{2}$ wöchentlich verabreicht, in Europa in einer Dosierung von $350 \mathrm{mg} / \mathrm{m}^{2}$ alle 3 Wochen. D ie E rgebnisse dieser Studien waren indes ganz ähnlich. D ie objektiven R emissionsraten lagen im Bereich von $13 \%$, darüber hinaus wurde bei $40-50 \%$ der Patienten eine Tumorstabilisierung erreicht. Das mediane progressionsfreie Intervall betrug $4 \mathrm{M}$ onate, die mediane Ü berlebenszeit 9- 9,5 M onate.

\section{Phase-III-Studien mit Irinotecan}

Im weiteren Verlauf wurden zwei prospektive, randomisierte Phase-III-Studien in der Second-line-Therapie durchgeführt.

\begin{tabular}{ll}
\hline KARGER & ○ 2000 S. K arger G mbH , Freiburg \\
$\begin{array}{l}\text { Fax +49 761 4520714 } \\
\begin{array}{l}\text { E-mail Information@K arger.de } \\
\text { www.karger.com }\end{array}\end{array}$ & A ccessible online at: \\
www.karger.com/journals/onk
\end{tabular}

PD Dr. C. Bokemeyer

$\mathrm{H}$ ämatologie und $\mathrm{O}$ nkologie

M edizinische U niversitätsklinik

Ottfried-M üller-Str. 10

D-72076 Tübingen (G ermany) 


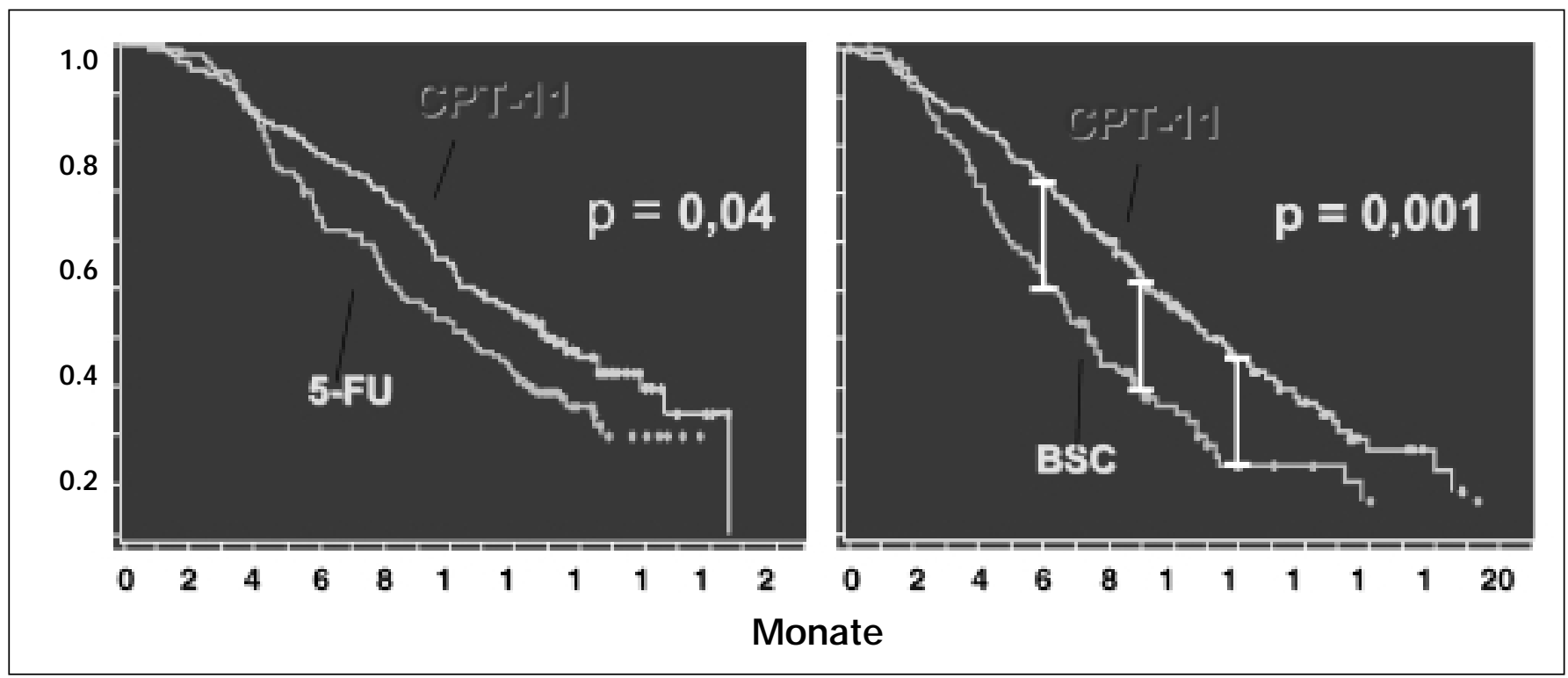

Abb.1 M edianes Ü berleben in den beiden Phase-III-Studien V 301 und V 302.In beiden Studien ergab sich ein signifikanter Ü berlebensvorteil zugunsten der Second-line-Therapie mit Irinotecan (mod. nach Cunningham et al. [2] und R ougier et al. [3]).

In der Studie V 301 mit insgesamt 279 Patienten wurde Irinotecan mit BSC verglichen [2], in der Studie V 302 (256 Patienten) mit einem 5-Fluorouracil-Standardschema; dabei konnten die teilnehmenden Zentren zwischen dem Schema von de $\mathrm{G}$ ramont, Lokich oder der A 10 wählen [3]. In beiden Studien waren die Patienten bereits mit 5-Fluorouracil vorbehandelt und hatten eine progrediente metastasierte Erkrankung. Primärer Endpunkt war jeweils die Überlebenszeit, sekundäre Endpunkte waren L ebensqualität, Verträglichkeit der Therapie, symptomfreies Intervall, progressionsfreies Intervall und Performance-Status.

D iese Studien zeigten, dass die Behandlung mit I rinotecan gegenüber der 5 -Fluorouracil-B ehandlung $(p=0,04)$ und noch deutlicher gegenüber BSC $(p=0,001)$ mit einem signifikanten Ü berlebensvorteil verbunden ist ( $A$ bb. 1 ). In der Vergleichsstudie versus BSC war die Ü berlebenswahrscheinlichkeit im I rinotecan-A rm nach 6 M onaten 1,4fach höher (72\% vs. 54\% ), nach 9 Monaten 1,8fach höher $(53 \%$ vs. $29 \%)$ und nach 12 M onaten sogar 2,6fach höher ( $36 \%$ vs. $14 \%$ ) [2]. Wichtigste Nebenwirkungen waren eine Neutropenie Grad $3 / 4$ bei 10-20\% der Patienten und eine verzögert einsetzende D iarrhö $\mathrm{G}$ rad $3 / 4$ bei $20-25 \%$ der Patienten.

\section{Nebenwirkungsmanagement}

$D$ iese N ebenwirkungen von I rinotecan muss man kennen, um sie adäquat behandeln zu können. Insbesondere bei gleichzeitigem A uftreten von N eutropenie und D iarrhö ist das I nfektionsrisiko deutlich erhöht, so dass eine frühzeitige Intervention nötig ist. E s hat sich allerdings in den bisherigen Studien gezeigt, dass mit zunehmender E rfahrung der behandelnden O nkologen im U mgang mit Irinotecan und durch eine sorgfältige U nterweisung der Patienten schwerwiegende Komplikationen im Zusammenhang mit der D iarrhö weitgehend ver- meidbar sind. A Is B ehandlungsmaßnahme hat sich hochdosiertes L operamid bewährt. D er Patient muss darüber informiert werden, dass er dieses A ntidiarrhoikum sofort bei einsetzender D iarrhö anzuwenden hat, und zwar sollte er alle 2 Stunden eine Dosis von $2 \mathrm{mg}$ einnehmen. Die Behandlung ist erst 12 Stunden nach Sistieren der Durchfälle zu beenden. $\mathrm{H}$ ält eine Diarrhö länger als 24 Stunden an, so ist insbesondere bei gleichzeitig bestehender Neutropenie eine orale A ntibiotikaprophylaxe mit einem Chinolonpräparat angezeigt. B esteht die D iarrhö auch nach 48 Stunden noch, so ist der betreffende Patient stationär aufzunehmen und intravenös zu rehydrieren. In jüngster Z eit wurden auch andere $M$ ethoden zur B ehandlung und Prophylaxe der verzögerten Diarrhö geprüft. So wurde nach oraler A nwendung des topischen Steroids Budesonid über eine deutliche B esserung der Diarrhö berichtet [4].

$D$ ie anderen Nebenwirkungen von Irinotecan sind ebenfalls gut beherrschbar. E in akutes cholinerges Syndrom tritt relativ häufig auf, in leichterer Form bei bis zu 50\% der Patienten. A usgeprägte Symptome sind selten ( $<5 \%$ der Patienten). B ei den betroffenen Patienten kommt es zu akuten Durchfällen, Schweißausbrüchen, Speichelfluss, Tränenfluss und anderen cholinergen Symptomen. A tropin S.c. (in der R egel 0,25 mg) hat sich als prophylaktische und therapeutische $\mathrm{Maßnnahme}$ bewährt. Wegen der relativ ausgeprägten emetischen Wirkung von I rinotecan gilt eine routinemäßige Prophylaxe mit 5- $\mathrm{H} \mathrm{T}_{3}^{-}$ A ntagonisten als Standard. A ndere vom 5-Fluorouracil bekannte Nebenwirkungen wie M ukositis, H auttoxizität usw. sind beim I rinotecan extrem selten.

\section{Einfluss auf die Lebensqualität}

A ngesicht der $\mathrm{N}$ ebenwirkungen, die mit jeder antineoplastischen Chemotherapie verbunden sind, wurde im $\mathrm{R}$ ahmen beider Phase-III-Studien die Lebensqualität der Patienten mit dem 
Fragebogen Q L Q - C 30 der E O R TC ausgewertet. Im Vergleich mit 5-Fluorouracil führte die Behandlung mit I rinotecan zu keiner B eeinträchtigung der L ebensqualität, im Vergleich mit B SC bewirkte I rinotecan sogar eine signifikante B esserung der $L$ ebensqualität. $D$ as bedeutet, dass der negative $E$ ffekt der $N$ ebenwirkungen durch die symptomatische B esserung bei der Tumorbehandlung durch I rinotecan mehr als kompensiert wird. Dies haben inzwischen auch neuere Studien bestätigt, deren E rgebnisse 1999 publiziert wurden. E ine große in D eutschland durchgeführte Studie an 321 Patienten, bei denen eine vorausgegangene 5-Fluorouracil-B ehandlung versagt hatte, ergab mit der Second-line-Therapie mit Irinotecan $350 \mathrm{mg} / \mathrm{m}^{2}$ alle 3 Wochen eine objektive A nsprechrate von 10\% [5]. D ie R ate der Tumorstabilisierungen (bei durchweg progredienter E rkrankung zu Studienbeginn) betrug jedoch $64 \%$. E in ähnliches E rgebnis wurde mit dem wöchentlichen Schema $\left(125 \mathrm{mg} / \mathrm{m}^{2} /\right.$ Woche) an 65 Patienten mit Fluorouracil-refraktärer E rkrankung (Progression/Rezidiv innerhalb von 6 Monaten nach 5-F luorouracil-Therapie) erzielt [6]. D ie objektive R emissionsrate betrug $10 \%$ und die $\mathrm{R}$ ate von Tumorstabilisierungen $45 \%$. 40,5\% der auswertbaren Patienten zogen einen palliativen N utzen aus der B ehandlung, d.h. Schmerzen oder PerformanceStatus besserten sich nachhaltig.

A us den vorliegenden $D$ aten geht somit durchweg hervor, dass der Prozentsatz der Patienten, die von der Behandlung mit I rinotecan in Form einer symptomatischen B esserung profitieren, um ein M ehrfaches höher ist als die objektive R emissionsrate. U mgekehrt bedeutet dies, dass eine radiologisch messbare Remission nicht die Voraussetzung ist, um eine B esserung der B eschwerden erwarten zu können.

\section{Aktuelle Entwicklungen}

$N$ ach wie vor gibt es jedoch eine R eihe noch nicht vollständig geklärter Fragen im Zusammenhang mit der Second-lineTherapie mit Irinotecan:

- Sollten alle Patienten nach Versagen einer 5-FluorouracilTherapie mit Irinotecan behandelt werden bzw. welche A uswahlkriterien bezüglich Performance-Status, voraussichtlicher Toleranz der Therapie oder A Iter sollten angelegt werden?

- Welches ist das wirksamste und sicherste IrinotecanSchema?

- Wie wirksam ist I rinotecan nach einer hochdosierten 5-Fluorouracil/Folinsäure-Therapie (AIO-Schema) in der Second- und Third-line-Therapie?

- Wie wirksam ist Irinotecan nach einer vorausgegangenen Kombinationstherapie mit 5-Fluorouracil/0 xaliplatin?

- Ist die Kombinationstherapie mit Irinotecan + Hochdosis5-Fluorouracil/Folinsäure bei vorausgegangener Progression unter dem A I O -Schema günstiger als eine I rinotecan-M onotherapie?

Z ur Frage der Wirksamkeit und Sicherheit des wöchentlichen I rinotecan-Schemas liegen mittlerweile $D$ aten einer neueren Phase-II-Studie aus den U SA vor [7]. 166 Patienten mit R ezidiv oder Progression nach einer 5-Fluorouracil-Behandlung erhielten D osen von entweder 125 oder $100 \mathrm{mg} / \mathrm{m}^{2} /$ Woche. Im Vergleich beider D osierungen zeigte sich zwar mit $125 \mathrm{mg} / \mathrm{m}^{2} /$
Woche eine geringfügig höhere G esamtansprechrate (14 vs. $9 \%$ ) und Stabilisierungsrate (44 vs. $38 \%$ ) als mit der niedrigeren D osierung, doch dieser U nterschied war nicht signifikant. Die Toxizität war bis auf vermehrtes Erbrechen Grad 3/4 ( 21 vs. $2 \%$ ) in der $125-\mathrm{mg} / \mathrm{m}^{2}$-G ruppe mit beiden D osierungen recht ähnlich. Somit ist das wöchentliche Schema sicherlich eine hilfreiche B ehandlungsoption, da sie die M öglichkeit gibt, die Patienten engmaschig zu beobachten und bei auftretender Toxizität gegebenenfalls eine wöchentliche A pplikation auszulassen. In dieser Studie wurde auch der Zusammenhang zwischen Alter und Verträglichkeit der Therapie genauer untersucht. D ie kumulative Häufigkeit einer D iarrhö G rad 3/4 war bei den älteren Patienten (über $65 \mathrm{~J}$ ahre) signifikant höher als bei den jüngeren (38 vs. $19 \%, p=0,008$ ). In Zukunft scheinen deshalb gezielte $U$ ntersuchungen in einem geriatrischen Patientengut sinnvoll zu sein, um auf die speziellen B edürfnisse und Probleme dieser A Itersgruppe im Rahmen der Behandlung des Kolorektalkarzinoms genauer eingehen zu können.

Die Frage der Wirksamkeit von Irinotecan $\left(350 \mathrm{mg} / \mathrm{m}^{2}\right.$ alle 3 Wochen) nach Vorbehandlung mit einem Hochdosis-5-Fluorouracil/Folinsäure-Schema wurde aktuell in zwei multizentrischen Studien in D eutschland untersucht. In der Studie von Schöffski et al. [8], deren E rgebnisse auf dem A SCO-M eeting 2000 vorgestellt werden, erhielten 107 Patienten I rinotecan in der Second-line-Situation nach Vorbehandlung mit dem A 10 Schema. Die zweite Studie behandelt Patienten in der Thirdline-Situation (nach First-line-Therapie mit dem MayoSchema und Second-line-Therapie mit dem A IO-Schema). In der Second-line-Situation wurde eine objektive Remissionsrate von $12 \%$ erreicht und bei weiteren $59 \%$ der Patienten eine Tumorstabilisierung. Die Häufigkeit einer Diarrhö Grad 3/4 betrug $23 \%$, die einer Neutropenie $16 \%$. In der Third-lineSituation zeichnen sich bei den in Tübingen behandelten Patienten sowohl in Bezug auf die Effektivität als auch die Toxizität ganz ähnliche E rgebnisse ab.

Inzwischen wurde in mehreren Studien in der Secondline-Therapie auch Irinotecan mit 0 xaliplatin, einer PlatinKomplexverbindung der 3. G eneration, kombiniert. In zwei in A bstractform publizierten Phase-I/II-Studien wurde ein 14-tägiges und ein 3-wöchentliches Schema eingesetzt $[9,10]$. A Is geeignet für Phase-II-Studien erwiesen sich 0 xaliplatin $85 \mathrm{mg} / \mathrm{m}^{2}+$ Irinotecan $200 \mathrm{mg} / \mathrm{m}^{2}$ alle 3 Wochen und Oxaliplatin $85 \mathrm{mg} / \mathrm{m}^{2}+$ I rinotecan $175 \mathrm{mg} / \mathrm{m}^{2}$ alle 2 Wochen. D osislimitierend waren die Neutropenie und die durch 0 xaliplatin hervorgerufene Polyneuropathie. In beiden Studien wurde eine hohe objektive R emissionsrate um $50 \%$ und eine Tumorstabilisierungsrate um $40 \%$ erzielt. In der bislang einzigen voll publizierten Studie wurden 36 Patienten nach Versagen einer 5-Fluorouracil/Folinsäure-Behandlung (d.h. mit Progression während oder innerhalb von $6 \mathrm{M}$ onaten nach Beendigung der Chemotherapie) mit dem folgenden Kombinationsschema behandelt: 0 xaliplatin $85 \mathrm{mg} / \mathrm{m}^{2}$, Tag 1 und 15 , und Irinotecan $80 \mathrm{mg} / \mathrm{m}^{2}$, Tag 1, 8 und 15, alle 4 Wochen mit G-CSF-Support [11]. M it diesem Schema war die gastrointestinale Toxizität dosislimitierend (19\% Diarrhö Grad 3/4). Die objektive A nsprechrate betrug $42 \%$ (einschließlich $6 \%$ kompletter Remissionen) und die Tumorstabilisierungsrate $36 \%$. Die mediane Ü berlebenszeit übersteigt 14 M onate. 
Auch zur Kosteneffektivität einer Second-line-Therapie mit I rinotecan liegen inzwischen einige $D$ aten vor. I $m$ R ahmen der weiter oben besprochenen randomisierten Vergleichsstudie von I rinotecan mit einem 5-Fluorouracil-Infusionsschema [3] wurde auch eine A nalyse unter Zugrundelegung britischer Kostendaten vorgenommen [12]. B erücksichtigt wurden die Kosten für M edikamente, Verabreichung, $M$ anagement von Nebenwirkungen und zusätzliche supportive Maßnahmen. $D$ abei ergaben sich für die B ehandlung mit I rinotecan lediglich M ehrkosten von 1500 EUR (12150 vs. 10600 EUR), die aber in Relation zu setzen sind zu dem signifikanten Ü berlebensgewinn. Demnach ist eine Second-line-Therapie mit I rinotecan nicht nur medizinisch sinnvoll, sondern auch aus ökonomischer Sicht.

\section{Zusammenfassung}

$N$ ach den umfangreichen E rfahrungen aus europäischen und amerikanischen Studien steht mit I rinotecan für die Second-
line-Therapie des fortgeschrittenen Kolorektalkarzinoms ein effektives M edikament zur Verfügung, dessen Nebenwirkungen bei entsprechender E rfahrung gut beherrschbar sind. I rinotecan induziert eine objektive Remissionsrate von etwa $13 \%$, darüber hinaus wird bei $40-50 \%$ der Patienten ein Stillstand des Tumorwachstums erreicht. $D$ ie mediane $D$ auer der R emissionen bzw. Tumorstabilisierungen liegt bei etwa $6 \mathrm{M} \mathrm{o-}$ naten, die mediane Ü berlebensdauer bei ungefähr $9 \mathrm{M}$ onaten. $D$ ie häufigsten Nebenwirkungen der B ehandlung sind Diarrhö, Ü belkeit und N eutropenie. Z wischen wöchentlichen und 3-wöchentlichen A pplikationsschemata besteht offenbar kein nennenswerter Wirkungsunterschied. M öglicherweise werden sich in Zukunft auch Kombinationen, Z.B. von I rinotecan mit O xaliplatin, für die Second-line-Therapie als sinnvoll erweisen. $D$ ies muss aber unter B erücksichtigung der $E$ ntwicklungen in der First-line-Therapie sorgfältig und differenziert beurteilt werden.

\section{Literatur}

1 de $G$ ramont $A$, B osset J F, Milan C, R ougier $P$, B ouche $O$, E tienne $P L, M$ orvan $F$, L ouvet $C, G$ uillot $T$, B edenne $\mathrm{L}: \mathrm{A}$ prospectively randomized trial comparing $5-\mathrm{FU}$ bolus with low dose folinic acid and 5-FU bolus plus continuous infusion with high dose folinic acid for advanced colorectal cancer. Proc A m Soc Clin O ncol 1995;14:455(abstr).

2 Cunningham D, Pyrhonen S, James RD, Punt CJ Hickish TF, Heikkila R, Johannesen TB, Starkhammar $H$, Topham CA , A wad L, J acques $C$, H erait $P$ : R andomised trial of irinotecan plus supportive care versus supportive care alone after fluorouracil failure for patients with metastatic colorectal cancer. L ancet 1998:352:1413-1418.

3 Rougier P, Van Cutsem E, Bajetta E, Niederle N, Possinger $K$, Labianca $R, N$ avarro $M, M$ orant $R$, B leiberg $\mathrm{H}$, Wils J, A wad L, H erait $P$, Jacques $C$ : $R$ andomised trial of irinotecan versus fluorouracil by continuous infusion after fluorouracil failure in patients with metastatic colorectal cancer. L ancet 1998; 352:1407-1412.

4 Lenfers BH, L oeffler TM, Droege CM , H ausamen TU: Substantial activity of budesonide in patients with irinotecan (CPT-11) and 5-fluorouracil induced diarrhea and failure of loperamide treatment. A nn Oncol 1999;10:1251-1253.
5 H oeffken K, Ridwelsky C, Wein A, M ezger J, Stoffregen $C$, Weber $B$, Fink $M, H$ emmers $H$ : Phase II study of irinotecan as second line chemotherapy (CT) in metastatic colorectal cancer (CR C). Proc A m Soc Clin O ncol 1999;18:937(abstr)

$6 \mathrm{M}$ ichael $M, M$ oore $M J$, Hedley $D, O$ za $A$, Feld $R$ G oel R, M aroun J, Jolivet J, Fields A : A phase II study of irinotecan (CPT-11) as palliative therapy in refractory advanced colorectal cancer (CR C). Proc A m Soc Clin O ncol 1999;18:934(abstr).

7 R othenberg ML, Cox JV, DeVore RF, H ainsworth JD, Pazdur R, R ivkin SE, M acdonald J S, G eyer CE, Sandbach J, Wolf DL, Mohrland JS, Elfring GL, M iller $\mathrm{LL}$, Von $\mathrm{H}$ off $\mathrm{DD}$ : A multicenter, phase II tria of weekly irinotecan (CPT-11) in patients with previously treated colorectal carcinoma. Cancer 1999;85:786-795.

8 Schöffski P, Vanhoefer U, Kirchner H, Trenn G, Bokemeyer C, Preusser P, A chterrath W, Deuß B, Wilke $\mathrm{H}$ : Phase II study of irinotecan as second line chemotherapy in metastatic colorectal cancer after prior exposure to infusional 5-FU-based chemotherapy. Proc A m Soc Clin Oncol 2000;19:1155 (abstr).
$9 \mathrm{G}$ oldwasser $F$, G ross $M$, Tigaud J $M$, Jasmin $C$ M arceau-Suissa J, M isset J L, C vitkovic E: CPT-11/ oxaliplatin (L-OH P) combination every two weeks: Final results of a phase I study in advanced digestive malignancies. Proc A m Soc Clin Oncol 1999;18: 675(abstr).

10 Wasserman $E$, Kalla S, Misset J L, Goldwasser $F$ B edairia N, B ensamine M A, M arty $M$, C vitkovic $E$ : Oxaliplatin (L-OH P) and irinotecan (CPT 11) phase I/II studies: $R$ esults in $5 \mathrm{FU}$ refractory (FR) colorectal cancer (CR C) patients (pts). Proc A m Soc Clin Oncol 1999;18:913(abstr).

11 Scheithauer W, Kornek G V, R aderer M, Valencak J, Weinlander G, Hejna M, H aider K, K wasny W, Depisch D: Combined irinotecan and oxaliplatin plus granulocyte colony-stimulating factor in patients with advanced fluoropyrimidine/leucovorinpretreated colorectal cancer. J Clin O ncol 1999;17: 902-906.

12 I veson TJ, Hickish T, Schmitt C, Van Cutsem E: I rinotecan in second-line treatment of metastatic colorectal cancer: I mproved survival and cost-effect compared with infusional 5-FU. Eur J Cancer 1999:35:1796-1804. 